\title{
Chronic lymphocytic leukemia and concurrent seminoma in the same testis
}

\author{
Kosuke Miyai $^{1,2}$, Fumihisa Kumazawa ${ }^{1}$, Kimiya Sato ${ }^{1}$, Hitoshi Tsuda ${ }^{1}$ \\ 'Department of Basic Pathology, National Defense Medical College, Saitama; ²Department of Pathology, Japan Self-Defense Forces Central Hospital, Tokyo, Japan
}

\begin{abstract}
A 59-year-old man presented with a painless testicular mass and underwent a radical orchiectomy. The resected specimen showed a 5-cm-sized, white-yellow and homogenous solid mass in the testicular parenchyma. Histologically, the central part of the tumor exhibited typical features of seminoma. The peripheral part of the tumor exhibited diffuse infiltration of small, monotonous lymphoid cells involving the tunica albuginea. The monotonous lymphoid cells were immunoreactive for CD20, CD79a, CD5, and CD23, and negative for CD3, CD10, and cyclin D1. Kappa light chain restriction was detected on flow cytometry using the resected specimen. Considering the circulating lymphoid cell count of $>5.0 \times 10^{3} / \mathrm{hL}$, we diagnosed the peripheral component of the tumor as an infiltration of chronic lymphocytic leukemia. This extremely rare combination of seminoma and lymphoid neoplasm should be considered in the differential diagnosis of classic seminoma with extensive lymphoid reaction in tumors arising in elderly patients.
\end{abstract}

Key Words: Testis; Seminoma; Chronic lymphocytic leukemia; Concurrent tumor

Received: May 31, 2021 Revised: July 12, 2021 Accepted: September 10, 2021

Corresponding Author: Kosuke Miyai, MD, PhD, Department of Basic Pathology, National Defense Medical College, 3-2 Namiki, Tokorozawa, Saitama 359-8513, Japan Tel: +81-4-2995-1211, Fax: +81-4-2996-5193, E-mail: mykusu228@nifty.com

Germ cell tumors derived from germ cell neoplasia in situ (GCNIS) account for more than $90 \%$ of testicular tumors, and approximately half of these tumors are histologically determined to be pure seminomas [1]. In contrast, less than $5 \%$ of testicular neoplasms are malignant lymphomas, most of which are diffuse large B-cell lymphomas (DLBCLs) [2]. As the distribution of age at onset differs between testicular germ cell tumors (20-45 years) and lymphomas (> 50 years) $[1,2]$, only a few reports have described the concurrent and/or synchronous presentation of testicular seminoma and lymphoid neoplasms [3-6]. Leukemia, including lymphoid and myelogenous subtypes, can also involve testis in a subset of cases $[7,8]$. Chronic lymphocytic leukemia (CLL) is an indolent type of lymphoid neoplasm, characterized by an increased number of peripheral monoclonal B-lymphocytes, as well as lymphadenopathy, organomegaly, and cytopenia. Extranodal involvement-including the skin, gastrointestinal tract, or central nervous system - has been reported in a small subset of the patients, and testicular involvement is relatively rare [7]. Recently, we experienced a unique case of CLL and concurrent seminoma in a single tumor of the same testis. Herein, we de- scribe the clinicopathological features of this case.

\section{CASE REPORT}

A 59-year-old Japanese man, without any notable past or familial medical history, was admitted to our hospital because of a painless right testicular mass. Abdominal and pelvic computed tomography (CT) revealed a $4.5 \mathrm{~cm}$-sized mass in the right testicular parenchyma without evidence of ascites or nodal/distant metastasis. His interleukin-2 receptor level was $613 \mathrm{U} / \mathrm{mL}$ (normal range, 122 to $496 \mathrm{U} / \mathrm{mL}$ ), and the levels of lactate dehydrogenase, $\alpha$-fetoprotein, and human chorionic gonadotropin were within the normal limits. His white blood cell count on admission was $12.0 \times 10^{3} / \mu \mathrm{L}$ with a lymphocyte percentage of $69.3 \%$ (absolute lymphocyte count, $8.3 \times 10^{3} / \mu \mathrm{L}$ ). No obvious anemia or thrombocytopenia was observed. Under the clinical diagnosis of a germ cell tumor or malignant lymphoma, right radical orchiectomy was performed.

The cut surface of the resected specimen showed a well-circumscribed, white-yellow and homogenous solid mass with no 
hemorrhage or necrosis measuring $5.0 \times 4.0 \times 2.9 \mathrm{~cm}$ in the testicular parenchyma (Fig. 1). Histologically, the tumor consisted of two components: a central multinodular component and a peripheral diffuse infiltrative component (Fig. 2A). The multinodular component was composed of large polygonal cells with clear cytoplasm and prominent nucleoli intermingled with lymphocytes (Fig. 2B). Around the nodular proliferation of polygonal tumor cells, dense infiltration of variously sized lymphocytes with multiple lymphoid follicles was observed. In contrast, the peripheral part of the tumor exhibited monotonous, diffuse infiltration of small lymphoid cells, focally involving the entire tunica albuginea (Fig. 2C). Immunohistochemically, the large polygonal cells were diffusely positive for octamer-binding transcription factor 4 (Oct- 4 ), and scattered Oct-4-positive cells were detected in the peritumoral seminiferous tubules (Fig. 2D); these observations confirmed the diagnosis of seminoma and the corresponding GCNIS. No vascular permeation, invasion beyond the tunica albuginea, or spermatic cord invasion by the seminoma cells was observed (pathological T1).

The peripheral monotonous lymphoid cells were immunoreactive for $\mathrm{CD} 20, \mathrm{CD} 79 \mathrm{a}, \mathrm{CD}$, and $\mathrm{CD} 23$ and negative for $\mathrm{CD} 3$, CD21, CD10, and cyclin D1 (Fig. 2E, F). Kappa light chain restriction was detected during flow cytometry using the resected specimen. Considering that the patient's circulating lymphoid cell count was $>5.0 \times 10^{3} / \mu \mathrm{L}$ at admission, the peripheral component of the tumor was diagnosed as testicular involvement of CLL. Immunostaining for CD21 revealed a retained follicular dendritic-cell meshwork in the lymphoid stroma of the seminoma (Fig. 3A). The Ki67 labeling index in the lymphoid stroma of the seminoma was much higher than that in CLL (approximately

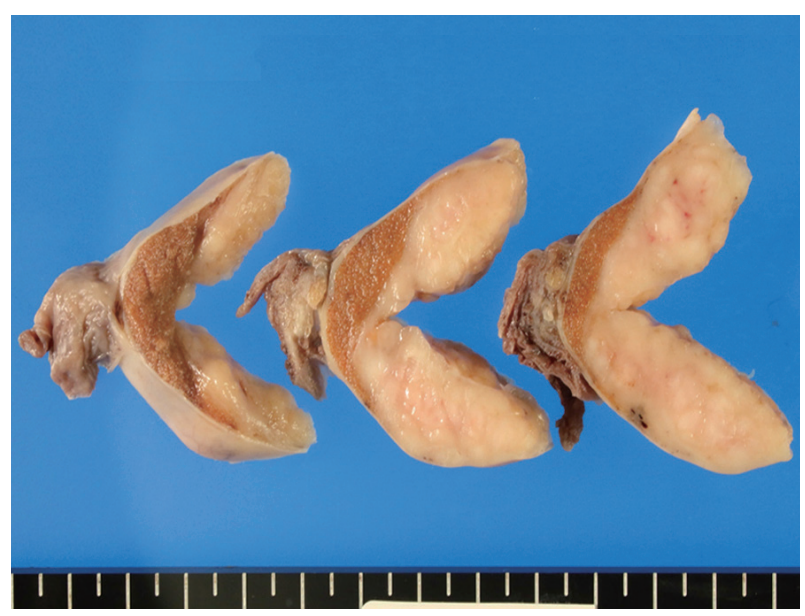

Fig. 1. The cut surface of the resected specimen shows a whiteyellow, solid mass measuring $5.0 \mathrm{~cm}$ in the right testicular parenchyma.
$30 \%$ vs. $<5 \%$ ) (Fig. 3B). These findings suggested that CLL was not intermixed with the lymphoid stroma of the seminoma; these two components existed exclusively within a single tumor mass. Postoperative fluorodeoxyglucose positron emission tomography/ CT showed no obvious nodal lesions or mass-forming lesions within systemic organs. The patient required no additional therapy during follow-up, and remained alive and well, with no evidence of disease progression, recurrence, or metastases after 2 years.

\section{DISCUSSION}

A vast majority of primary testicular lymphoid neoplasms (80\%-95\%) are DLBCLs [2]. Previously reports of other rare histological types of malignant lymphoma included mantle cell lymphoma [9], testicular follicular lymphoma [10], extranodal marginal zone lymphoma [11], natural killer/T-cell lymphoma, nasal type [12], peripheral T-cell lymphoma (PTCL) [13], and activin receptor-like kinase-1-negative anaplastic large cell lymphoma [14]. On the other hand, testicular involvement of lymphoid leukemia has been described in some previous reports $[7,8]$. In a previous report reviewing 13,500 autopsy and 641 surgical specimens, seven cases with lymphoid leukemia secondarily involving the testis were detected; 6 were acute lymphoblastic leukemia (without information of B- or T-cell linage) and only one case was CLL [7]. Schniederjan and Osunkoya [8] identified 40 patients with lymphoid neoplasms of male urogenital organs. Of these, two were acute B-lymphoblastic leukemia in the testis and 4 were CLL in the prostate [8]. There have been a few reported cases of Richter syndrome, defined as the development of DLBCL in a patient with a previous or concomitant CLL/small lymphocytic lymphoma, with testicular involvement $[15,16]$. In the present report, we described an additional rare case of testicular involvement of CLL without progression to DLBCL.

The present case also showed the synchronous, concurrent CLL and seminoma in a single testicular tumor. There is no obvious pathophysiologic link or common risk factor between seminoma and CLL. Our literature review found a few rare examples of patients with synchronous seminoma and lymphoid neoplasms. All of these patients-including the present one-were aged more than 50 years [3-6]. Singh et al. [3] reported a case of synchronous classical Hodgkin lymphoma in the mediastinal/intraabdominal lymph nodes and right testicular seminoma. In another case, a huge pelvic mass and partially obstructed small intestine were simultaneously, but separately, resected, and histological examination of these two lesions revealed pure seminoma and DLBCL, respectively [4]. Jacobsen et al. [5] described 

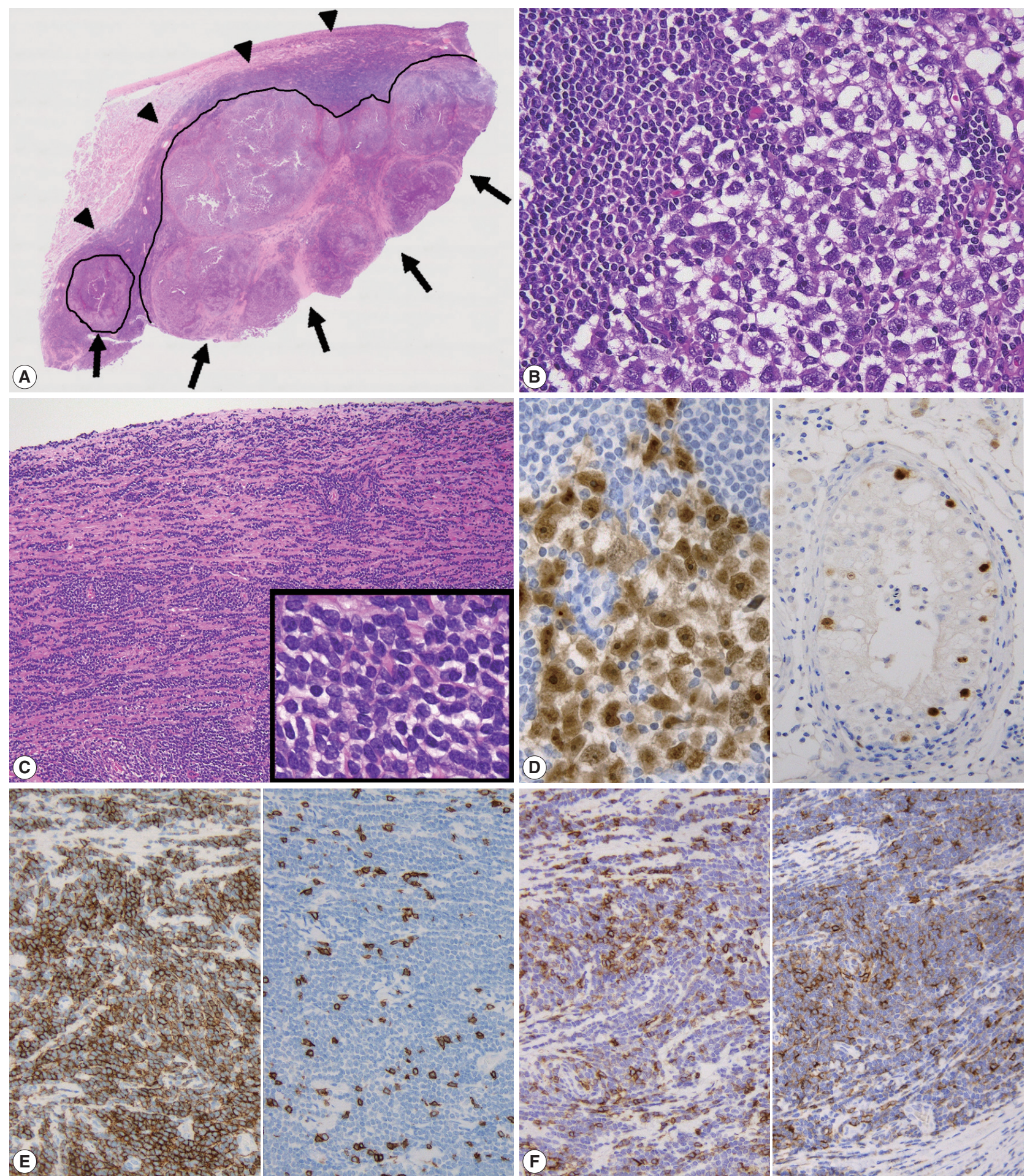

Fig. 2. (A) The scan image showing a central multinodular component (arrows) and peripheral diffuse infiltrative component (arrowheads). (B) The multinodular component comprises large polygonal cells with clear cytoplasm and prominent nucleoli intermingled with variously sized lymphocytes. (C) The peripheral component showing diffuse, dense infiltration of small lymphoid cells involving the entire tunica albuginea. Lymphoid cells with hyperchromatic and monotonous nuclei are noted (inset). (D) The large polygonal cells are diffusely positive for octamerbinding transcription factor 4 (Oct-4) (left), and scattered Oct-4-positive cells are detected in the seminiferous tubules (right). (E, F) Small, monotonous lymphoid cells are diffusely immunoreactive for CD20 (E, left), CD5 (F, left), and CD23 (F, right) and negative for CD3 (E, right). 

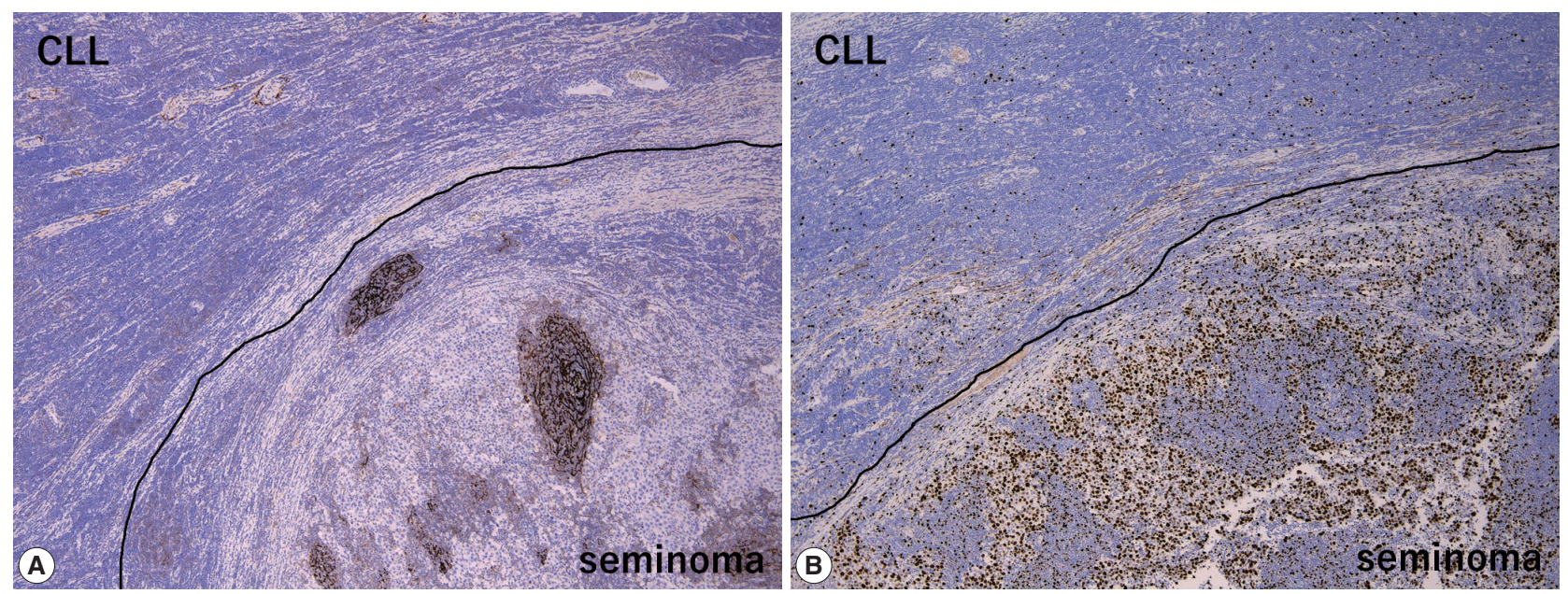

Fig. 3. (A) Meshwork of CD21+ follicular dendritic cells is retained in the lymphoid stroma of the seminoma; it is absent in the chronic lymphocytic leukemia (CLL). (B) The Ki-67 labeling indices in the lymphoid stroma of the seminoma and CLL were approximately $30 \%$ and $<5 \%$, respectively.

a case of metastatic seminoma and concurrent follicular lymphoma, grade 1 in a supraclavicular lymph node. Only one case of concurrent seminoma and PTCL, NOS in the same testis has been reported [6]. Because of indolent clinical courses of CLL and seminoma, additional/adjuvant therapy was not administered for both tumors in the present case. However, concurrent management of the two different malignancies is usually necessary in these cases. An accurate pathological diagnosis is especially important for improving the patients' clinical courses.

Concerning the large cell component of the present tumor, the presence of GCNIS and careful histological/immunohistochemical examination made it possible to distinguish the seminoma from other tumors including large cell lymphoid neoplasms (DLBCL, PTCL, and anaplastic large cell lymphoma) and solidtype non-seminomatous tumors (embryonal carcinoma and yolk sac tumor). However, when DLBCL is detected, surrounded by dense, small lymphocytic infiltration as in the present case, Richter syndrome should be considered during differential diagnosis $[15,16]$. On the other hand, differential diagnoses of the small cell component of the present tumor (i.e., CLL) include non-neoplastic, reactive lymphocytes and other mature small B-cell lymphomas, such as follicular lymphoma, mantle cell lymphoma, and extranodal marginal zone lymphoma. Of these, the most important differential diagnosis is small reactive lymphocytes infiltrating into the seminoma, which can be misdiagnosed as "twocell pattern" of classic seminoma. Compared to small monotonous lymphoid tumor cells of CLL, lymphoid stroma intervening in seminoma cells is composed of various sizes of lymphocytes. In addition, reactive lymphocytes in seminoma sometimes exhibit lymphoid follicles with CD21-positive follicular dendritic-cell meshwork (Fig. 3A) and show much higher Ki67 labeling index than CLL (Fig. 3B). Finally, because immunohistochemical detection of the monoclonality of lymphoid cells without plasmacytic differentiation is sometimes challenging, light chain restriction detected by flow cytometry is useful for ruling out nonneoplastic, reactive lymphocytic infiltration. Our patient's specific immunohistochemical findings (CD20+, CD $3+$, and $\mathrm{CD} 23+$ ) and a circulating lymphoid cell count of $>5.0 \times 10^{3} / \mu \mathrm{L}$ supported the diagnosis of CLL rather than that of other mature small B-cell lymphomas.

In summary, we have described a case of isolated CLL with concurrent seminoma in the same testis. With respect to diagnostic and therapeutic aspects, this extremely rare combination should be kept in mind for both clinicians and pathologists.

\section{Ethics Statement}

This single case report in exempted submission to Institutional Review Board and subsequent informed consent by the National Defense Medical College, Tokorozawa, Japan.

\section{Availability of Data and Material}

Data sharing not applicable to this article as no datasets were generated or analyzed during the study.

\section{Code Availability}

Not applicable.

\section{ORCID}

Kosuke Miyai https://orcid.org/0000-0003-1776-3541

Fumihisa Kumazawa https://orcid.org/0000-0001-9019-669X

Kimiya Sato https://orcid.org/0000-0002-8534-8852 
Hitoshi Tsuda https://orcid.org/0000-0003-3457-4224

\section{Author Contributions}

Conceptualization: KM. Data curation: KM, FK. Formal analysis: KM. Funding acquisition: KM. Investigation: KM, FK, KS. Methodology: KM, FK, KS. Resources: KS, HT. Supervision: KS, HT. Visualization: KM. Writing_original draft: KM. Writing — review \& editing: FK, KS, HT. Approval of final manuscript: all authors.

\section{Conflicts of Interest}

The authors declare that they have no potential conflicts of interest.

\section{Funding Statement}

This work was supported by JSPS KAKENHI Grant Number 19K16729 (KM).

\section{Acknowledgments}

The authors deeply appreciate Drs. Shojiro Morinaga and Koichi Oshima for their advice regarding the pathological diagnosis of the present case.

\section{References}

1. Ulbright TM, Amin MB, Balzer B, et al. Germ cell tumours. In: Mock H, Humphrey PA, Ulbright TM, Reuter VE, eds. WHO classification of tumours of the urinary system and male genital organs. 4th ed. Lyon: IARC Press, 2016; 189-226.

2. Cheah CY, Wirth A, Seymour JF. Primary testicular lymphoma. Blood 2014; 123: 486-93.

3. Singh P, Toom S, Shrivastava M, et al. Synchronous Hodgkin's lymphoma and seminoma: a rare coexistence and an important lesson. Clin Case Rep 2017; 5: 1658-9.

4. White MA, Dehaan AP, Wilson JM, Zakem MH, Maatman TJ. Synchronous advanced pure seminoma and diffuse large B-cell lymphoma: a case of multiple oncologic dilemmas. J Clin Oncol 2009; 27: e181-3

5. Jacobsen E, Chen JH, Schurko B, Benson C, Oh WK. Metastatic seminoma and grade 1 follicular lymphoma presenting concurrent- ly in a supraclavicular lymph node: a case report. Cases J 2009; 2: 7273.

6. Kitagawa J, Goto N, Shibata Y, et al. Peripheral T-cell lymphoma, not otherwise specified and concurrent seminoma in testis. J Clin Exp Hematop 2015; 55: 169-74.

7. Dutt N, Bates AW, Baithun SI. Secondary neoplasms of the male genital tract with different patterns of involvement in adults and children. Histopathology 2000; 37: 323-31.

8. Schniederjan SD, Osunkoya AO. Lymphoid neoplasms of the urinary tract and male genital organs: a clinicopathological study of 40 cases. Mod Pathol 2009; 22: 1057-65.

9. Epstein AS, Hedvat C, Habib F, Hamlin P. Testis-isolated mantle cell lymphoma: a unique case. Clin Lymphoma Myeloma Leuk 2011; 11: 439-41.

10. Finn LS, Viswanatha DS, Belasco JB, et al. Primary follicular lymphoma of the testis in childhood. Cancer 1999; 85: 1626-35.

11. Kuper-Hommel MJ, Janssen-Heijnen ML, Vreugdenhil G, et al. Clinical and pathological features of testicular diffuse large B-cell lymphoma: a heterogeneous disease. Leuk Lymphoma 2012; 53: 242-6.

12. Liang DN, Yang ZR, Wang WY, et al. Extranodal nasal type natural killer/T-cell lymphoma of testis: report of seven cases with review of literature. Leuk Lymphoma 2012; 53: 1117-23.

13. Jun HJ, Kim WS, Yang JH, et al. Orbital infiltration as the first site of relapse of primary testicular T-cell lymphoma. Cancer Res Treat 2007; 39: 40-3.

14. Lagmay J, Termuhlen A, Fung B, Ranalli M. Primary testicular presentation of ALK-1-negative anaplastic large cell lymphoma in a pediatric patient. J Pediatr Hematol Oncol 2009; 31: 330-2.

15. Jha B, Dass J, Sachdev R, Bhargava R. Testicular swelling: a rare manifestation of chronic lymphocytic leukemia presenting with Richter's syndrome. Indian J Pathol Microbiol 2014; 57: 133-5.

16. Kawase K, Naiki T, Naiki-Ito A, et al. Rare case of Richter syndrome with testicular involvement successfully obtained good prognosis with rapid operation and immunochemotherapy. IJU Case Rep 2019; 2: 232-5. 